\title{
A Rare Case of Brugada Syndrome of a Female Patient Presented for Labour: Structured Anesthetic and Analgesic Plan for Delivery Management
}

\author{
Patrick Charles Thornton ${ }^{1,2}$, Mohamad Atef Radwan ${ }^{1}$
}

\section{Abstract}

32 Years old Primigravida, with Brugada syndrome presented for analgesic and anesthetic management for normal vaginal delivery which progressed to emergency caesarean section. We are presenting a structured approach aiming for avoiding medication that can trigger malignant arrhythmia; Possibly achieving minimal local anesthetic serum concentration which could trigger such attacks.

Key Words: Brugada syndrome; Combined spinal epidural; Local anaesthesia.

\section{Introduction}

Brugada syndrome is autosomal dominant arrhythmic disorder associated with a risk of ventricular fibrillation and sudden cardiac death in a structurally normal heart [1]. Although Brugada has more aggressive clinical course in male more than female, some reports suggested that the pregnancy could be one of arrythmia triggering factors [2].

We are presenting anesthetic and analgesic management of a laboring 32-year-old primigravida patient, suffered from Brugada syndrome with a family history of sudden arrhythmic death syndrome (SADS).

The Patient was referred to anesthesia clinic for planning of labor anesthesia and analgesia. After taking a detailed history and reviewing the cardiology consultation; she was found to have Brugada syndrome with type three changes on her resting ECG [3] (ST-segment elevation $<1 \mathrm{~mm}$ in $\mathrm{V} 1$ with incomplete right bundle branch block), with average corrected QT interval of $450 \mathrm{~ms}$. Family history revealed sudden arrhythmic death syndrome (SADS), for which she had a positive Ajmalin test in the context of family screening. Upon a complaint of palpitation episodes, she had an Implantable loop recorder which did not demonstrate any malignant arrythmia.

Brugada syndrome is autosomal dominant arrhythmic disorder associated with a risk of ventricular fibrillation and sudden cardiac death in a structurally normal heart. Although Brugada has more aggressive clinical course in male more than female, some reports suggested that the pregnancy could be one of arrythmia triggering factors.

We are presenting anesthetic and analgesic management of a laboring 32-year-old primigravida patient, suffered from Brugada syndrome with a family history of sudden arrhythmic death syndrome (SADS).

The Patient was referred to anesthesia clinic for planning of labor anesthesia and analgesia. After taking a detailed history and reviewing the cardiology consultation; she was found to have Brugada syndrome with type three changes on her resting ECG [3] (ST-segment elevation $<1 \mathrm{~mm}$ in $\mathrm{V} 1$ with incomplete right bundle branch block), with average corrected QT interval of $450 \mathrm{~ms}$. Family history revealed sudden arrhythmic death syndrome (SADS), for which she had a positive Ajmalin test in the context of family screening. Upon a complaint of palpitation episodes, she had an Implantable loop recorder which did not demonstrate any malignant arrhythmia. After a detailed discussion, Remifentanil patient-controlled analgesia (PCA) as a starting strategy was planned for the labor analgesia, with a step-up option towards combined spinal epidural analgesia (CSE) if satisfaction regarding pain control is not attained. For avoidance of local anesthetic hazardous effect on cardiac sodium channels which may be associated with a bolus regimen; CSE technique would be used with plain Bupivacaine $(1 \mathrm{ml})$ of $0.25 \%$ intrathecal and maintenance on continuous infusion protocol $(9.3 \mathrm{ml} /$ hour of $0.125 \%$ of levobupivacaine with $2 \mathrm{mcg}$ fentanyl/ml) per hospital protocol.

If Lower segment Cesarean section (LSCS) is required.; Epidural catheter is to be removed, and a standard spinal anesthesia is to be performed using initiation protocol of spinal anesthesia (2.2 to $2.4 \mathrm{ml}$ of heavy Bupivacaine $0.5 \%$ with $20 \mathrm{mcg}$ fentanyl and $100 \mathrm{mcg}$ of preservative free morphine).

On the day of the delivery, the patient was admitted, and anesthetic team was called for providing early analgesia. After attaching the patient to ASA standard monitoring (3 leads ECG with lead II monitoring, Pulse oximetry, and Noninvasive blood pressure), Remifentanil Patient controlled analgesia (PCA) started; (40 mcg bolus with 3 minutes lock out) with supplemental Oxygen via nasal prongs (2-4 liters). After 3 boluses she was satisfied.

Three hours later; category one (CAT I) Cesarean section was called for fetal
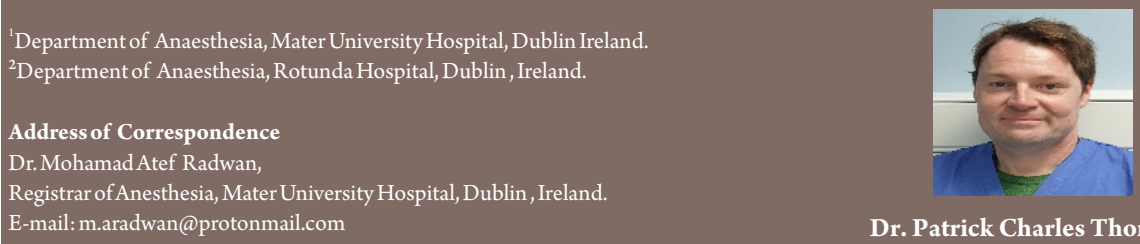

Dr. Patrick Charles Thornton

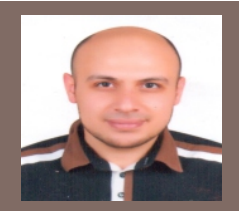

Dr. Mohamad Atef Radwan

(C) 2020 by Journal of Anaesthesia and Critical Care Case Reports| Available on www.jaccr.com | DOI: 10.13107/jaccr.2020.v06i02.151 This is an Open Access article distributed under the terms of the Creative Commons Attribution Non-Commercial License (http://creativecommons.org/licenses/by-nc/3.0) which permits unrestricted non-commercial use, distribution, and reproduction in any medium, provided the original work is properly cited. 


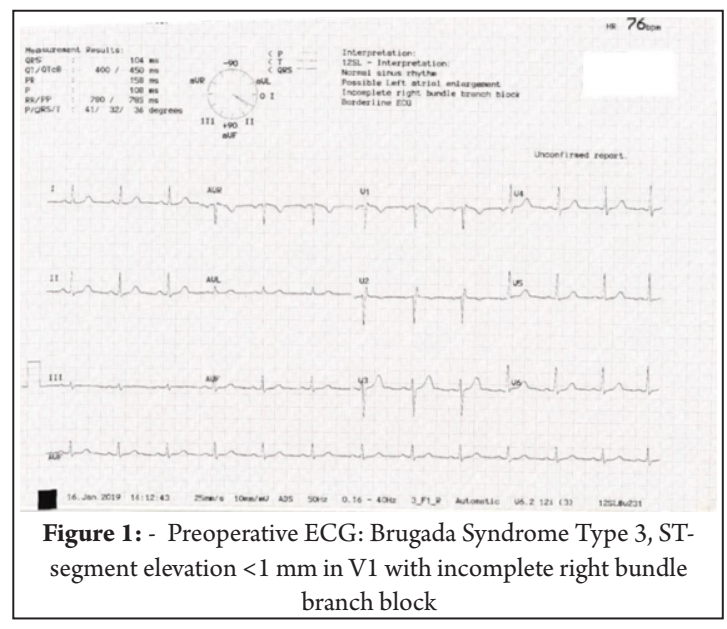

bradycardia which had recovered. Theatre team was prepared for doing general anesthesia. After a discussion with obstetric team concerning baby's safety, it was agreed to do spinal anesthesia under CTG monitoring. Rapid sequence spinal anesthesia [4] was performed using $2.4 \mathrm{ml}$ of heavy Bupivacaine without opioid, which achieved complete lower limb motor block with T4 sensory level block to the sharp and ice stimulation. Two incremental doses of $50 \mathrm{mcg}$ of phenylephrine were used for mild hypotension followed spinal anesthesia.

After baby's delivery, 5 Units intravenous infusion were given over 15 minutes for attenuating any possible cardiac arrhythmogenic effect. Additional Oxytocin infusion at rate 83 milliunit/min was used for postpartum hemorrhage prophylaxis. For post-operative drug prescription; Ondansetron was not prescribed. Oxycodone to a maximum dose of $60 \mathrm{mg} /$ day (with a restriction instruction to limit the dose to a maximum ts of $30 \mathrm{mg}$ per day if she will be breastfeeding). Non-steroidal antiinflammatory and paracetamol were prescribed for post-operative pain control. Cesarean section was finished uneventfully with average blood loss. Patient was transported to the recovery unit and observed under continuous ECG, Noninvasive blood pressure, and Pulse oximeter monitoring. Three days later, patient discharged from hospital uneventfully.
Discussion

Due to the rarity of Brugada syndrome ( 5 in 10,000 of the population) [5], it's anaesthetic management in a parturient patient may be challenging in a situation that local anaesthetic administration can be detrimental on cardiac conduction system. we presented a stepwise approach aimed to achieve a minimum serum local anaesthetic concentration. With a starting point of which Remifentanil may be used as a safer alternative for labour analgesia.

Remifentanil has an increasing popularity in usage as a second labour analgesic [6-8]. It is considered as a safe drug in Brugada syndrome [7,9]. It offers a safe and reliable analgesic option for labour in Brugada syndrome patients. It is not however, suitable for anaesthesia as a solo agent for operative delivery and our plan considered the possibility of the surgical delivery for this patient; Performing spinal anaesthesia and ensuring negligible amounts of bupivacaine in serum have been supported by many case reports [8-10].

Although Bupivacaine and propofol are among the drugs to be avoided for Brugada syndrome patients, however It has a "conflicting evidence and/or divergence of opinion about the drug, but the weight of evidence/opinion is in favour of a potentially arrhythmic effect in Brugada syndrome patients" [11]. Based on the case reports [9], we decided to use them in case of need, ensuring a low serum concentration of local anaesthesia whatever possible.

Whilst we did not need to use a CSE for our patient as remifentanil provided her with excellent labour analgesia; we believe that it might be a valid tool if employed sensibly. Minimizing serum concentration of bupivacaine by utilizing the 90 minutes labour analgesia or so of intrathecal administration of bupivacaine, then using of a low concentration $(0.125 \%)$ continuous infusion of levobupivacaine (with its safer cardiac profile) with avoiding of Programmed
Intermittent Epidural Boluses (PIEB); which may increase serum concentrations with the rapid bolus of local anaesthetics administration.

In this case we have not been exposed to preplanned (CSE) due to emergency situation, however we successfully used spinal anaesthesia as a solo agent for neuraxial block (2.4 of heavy Bupivacaine).

Regarding uterotonic drugs; Oxytocin was used softly via slow infusion rate, which did not result in any noticeable arrythmia. Ergometrine and Carboprost were discouraged by obstetric team for a possible fever and coronary spasm.

Patient's corrected QT was 450 milliseconds which is considered normal for post pubertal females [12], however, for extra precaution, we avoided the drugs which may be implicated in prolongation of QT. Ondansetron (though it is not contraindicated in Brugada) was omitted from post-operative drugs and Oxycodone was prescribed as a dose of less than 100 $\mathrm{mg} /$ day [13].

Of note, our patient did not have an Implantable cardioverter-defibrillator (ICD) in situ however this is a possibility for her in the future and many patient with Brugada syndrome will have ICDs and their management in labour and delivery must be considered.

\section{Conclusion}

In parturient with Brugada Syndrome, Remifentanil was used as an initial safe strategy for labor analgesia. Spinal anesthesia was used uneventfully in our patient and in many more case reports, which fosters the thought that the international community of obstetric anesthesia may consider advocating its careful use in this cohort of patient as a reasonably sage option for operative delivery. Oxytocin did not result in any form arrythmia when it was given slowly. Additional research may be needed regrading appropriate dose for epidural analgesia, in cases of continuous infusion, or incremental boluses.

\section{References}

1. Sorajja D, Ramakrishna H, Poterack A, Shen W-K, Mookadam F. Brugada syndrome and its relevance in the perioperative period. Ann Card Anaesth. 2015;

2. Giambanco L, Incandela D, Maiorana A, Alio W, Alio L. Brugada Syndrome and Pregnancy: Highlights on Antenatal and Prenatal Management. Case Rep Obstet Gynecol.2014;
3. Management of patients with a Brugada ECG pattern [Internet]. [cited 2019 Dec 1]. Available from: https://www.escardio.org/Journals/E-Journal-of-Cardiology-Practice/Volume$7 / M a n a g e m e n t-o f-p a t i e n t s-w i t h-a-B r u g a d a-E C G-p a t t e r n$, https://www.escardio.org/Journals/E-Journal-of-Cardiology-Practice/Volume7/Management-of-patients-with-a-Brugada-ECG-pattern 
4. Bhattacharya S, Ghosh S, Chattopadhya U, Saha D, Bisai S, Saha M. Rapid sequence spinal anesthesia versus general anesthesia: A prospective randomized study of anesthesia to delivery time in category-1 caesarean section.J Obstet Anaesth Crit Care. 2016Jul 1;6(2):75.

5. Brugada Syndrome [Internet]. NORD (National Organization for Rare Disorders). [cited 2019 Jun 9].Availablefrom: https://rarediseases.org/rare-diseases/brugada-syndrome/

6. Ohashi Y, Baghirzada L, Sumikura H, Balki M. Remifentanil for labor analgesia: a comprehensivereview. JAnesth. 2016;30(6):1020-30.

7. Martorano PP, Barboni E, Buscema G, Di Rienzo A. A Novel Anaesthetical Approach to Patients with Brugada Syndrome in Neurosurgery. Case Rep Anesthesiol. 2013;2013:1-3.

8. Choi YS, Lee WK, Lee HM, Min JH, Kim YH, Chae YK, et al. Spinal Anesthesia of a Patient with Brugada Syndrome: A case report. Korean J Anesthesiol. 49(4):574-7.

9. Staikou C, Chondrogiannis K, Mani A. Perioperative management of hereditary arrhythmogenic syndromes. BJA Br JAnaesth.2012 May 1;108(5):730-44.

10. Raval C, Saeed K. Anaesthetic management of a patient of Brugada syndrome for an emergency appendicectomy. Anesth Essays Res. 2012;6(1):101-4.

11. BrugadaDrugs.org | Safe drug use and the Brugada syndrome [Internet]. [cited 2019 Jun 3]. Availablefrom: https://www.brugadadrugs.org/

12. Crotti L, Celano G, Dagradi F, Schwartz PJ. Congenital long QT syndrome. Orphanet J Rare Dis. 2008 Jul 7;3:18.

13. Fanoe S, Jensen GB, Sjøgren P, Korsgaard MPG, Grunnet M. Oxycodone is associated with dosedependent $Q T c$ prolongation in patients and low-affinity inhibiting of $h E R G$ activity in vitro. BrJClin Pharmacol.2009 Feb;67(2):172-9.
Conflict of Interest: Nil

Source of Support: None
How to Cite this Article

Thornton P C, Radwan M A | A Rare Case of Brugada Syndrome of a Female Patient Presented for Labour: Structured Anesthetic and Analgesic Plan for Delivery Management |Journal of Anaesthesia and Critical Care Case Reports | May-August 2020; 6(2): 20-22. 